\section{Instability of Fixed, Low-Thrust Drag Compensation}

\author{
Colin R. McInnes* \\ University of Glasgow, \\ Glasgow, Scotland G12 8QQ, United Kingdom
}

\section{Introduction}

$\mathbf{F}$ ORCED drag compensation using continuous low-thrust propulsion has been considered for satellites in low Earth orbit. This simple, but nonoptimal, scheme merely requires that the thrust vector is directed opposite to the drag vector and that the magnitude of the two are equal. In principle, the drag force acting on the spacecraft could be determined onboard using accurate accelerometers. However, for small, low-cost spacecraft such sensors may be unavailable. An alternative strategy would be to fix the thrust magnitude equal to the expected air drag that would be experienced by the spacecraft. The thrust level would be periodically updated based on ground-based orbit determination. In this Engineering Note, it is shown that such a forced circular orbit with a fixed thrust level is exponentially unstable for all physically reasonable atmosphere models.

\section{Forced Drag Compensation}

For a circular low Earth orbit, the dominant perturbing effects are due to atmospheric drag and Earth oblateness. ${ }^{1}$ Atmospheric drag perturbations result in a secular decrease in orbital radius $r$ due to frictional energy loss. To fix the orbit radius, one possible scheme is to compensate directly for air drag $\boldsymbol{D}$ by applying an opposite, continuous low thrust $\boldsymbol{T}$. The equation of motion for a spacecraft of mass $m$ is then

$$
\ddot{\boldsymbol{r}}+\mu\left(\boldsymbol{r} / r^{3}\right)=(1 / m)(\boldsymbol{T}-\boldsymbol{D})
$$

which shows that if $\boldsymbol{T}=\boldsymbol{D}$ an ideal two-body orbit will be obtained, where $\mu$ is the gravitational parameter of the problem. When the scalar product of Eq. (1) is taken with the spacecraft velocity vector $v$, it is found that

$$
\frac{\mathrm{d}}{\mathrm{dt}}\left[\frac{1}{2} \boldsymbol{v} \cdot \boldsymbol{v}-\frac{\mu}{r}\right]=\frac{1}{m}(\boldsymbol{T}-\boldsymbol{D}) \cdot \boldsymbol{v}
$$

where the term in brackets of the left side is just the total orbit energy $E$. For a circular orbit, the total orbit energy is $E=-\mu / 2 r$. In addition, the local circular orbit speed $\|v\|=\sqrt{ }(\mu / r)$ so that

Received 15 July 2002; revision received 19 February 2003; accepted for publication 19 February 2003. Copyright (C) 2003 by Colin R. McInnes. Published by the American Institute of Aeronautics and Astronautics, Inc., with permission. Copies of this paper may be made for personal or internal use, on condition that the copier pay the $\$ 10.00$ per-copy fee to the Copyright Clearance Center, Inc., 222 Rosewood Drive, Danvers, MA 01923; include the code 0731-5090/03 \$10.00 in correspondence with the CCC.

*Professor, Department of Aerospace Engineering; colinmc@ aero.gla.ac.uk.
Eq. (2) may be used to obtain

$$
\frac{\mathrm{d} r}{\mathrm{~d} t}=\frac{2 r^{\frac{3}{2}}}{\sqrt{\mu}} \frac{\|\boldsymbol{T}-\boldsymbol{D}\|}{m}
$$

The general form of the air drag acting on the spacecraft is given by

$$
\|\boldsymbol{D}\|=\frac{1}{2} C_{D} A \rho(r) \boldsymbol{v} \cdot \boldsymbol{v}
$$

where $A$ is the aerodynamic reference area, $C_{D}$ is the drag coefficient, and $\rho$ is the air density at orbit radius $r$. Again when it is noted that $\|\boldsymbol{v}\|=\sqrt{ }(\mu / r)$ and constant thrust $\|\boldsymbol{T}\|$ is assumed, the circular orbit radius evolves according to

$$
\frac{\mathrm{d} r}{\mathrm{~d} t}=\frac{2\|\boldsymbol{T}\|}{\sqrt{\mu} m} r^{\frac{3}{2}}-\frac{\sqrt{\mu} C_{D} A}{m} \rho(r) r^{\frac{1}{2}}
$$

For a given operating orbit radius $\tilde{r}$, the required thrust to provide equilibrium is then given by

$$
\|\boldsymbol{T}\|=\left(C_{D} A \mu / 2 \tilde{r}\right) \rho(\tilde{r})
$$

With use of this fixed thrust, the stability of the resulting forced circular orbit will be investigated by linearization. Note that the forced circular orbit is in fact nonoptimal in terms of fuel consumption. ${ }^{2}$

\section{Linear Orbit Instability}

To determine the stability of the equilibrium operating orbit, the spacecraft orbit radius will be written as $r=\tilde{r}+\xi$. Then, expanding Eq. (5) to first order, with the required thrust provided by Eq. (6), yields

$$
\frac{\mathrm{d} \xi}{\mathrm{d} t}=\Lambda(\tilde{r}) \xi
$$

where the constant coefficient $\Lambda(\tilde{r})$ is found to be

$$
\Lambda(\tilde{r})=\left(\sqrt{\mu} C_{D} A / m\right)\left[\rho(\tilde{r}) / \tilde{r}^{\frac{1}{2}}\right]\left\{1-\tilde{r}\left[\rho^{\prime}(\tilde{r}) / \rho(\tilde{r})\right]\right\}
$$

and where $\rho^{\prime}(r)=\mathrm{d} \rho / \mathrm{d} r$. The condition required for stability is that $\Lambda(\tilde{r}) \prec 0$, which in turn requires that

$$
\rho^{\prime}(\tilde{r}) / \rho(\tilde{r}) \succ 1 / \tilde{r}
$$

If an exponential atmosphere is now assumed with scale height $H$ and base density $\rho_{0}$, so that

$$
\rho(r)=\rho_{0} \exp [-(r-R) / H]
$$

where $R$ is the radius of the Earth, the condition for stability then becomes

$$
-1 / H \succ 1 / \tilde{r}
$$

Because $\tilde{r} \succ 0$, this then implies that $H \prec 0$, which is clearly unphysical. In the general case, if $\rho^{\prime}(r) \sim \Delta \rho / \Delta r$, then Eq. (9) implies that

$$
\Delta \rho / \rho \succ \Delta r / \tilde{r}
$$

so that if $\Delta r \succ 0$, stability requires $\Delta \rho \succ 0$, which again is clearly unphysicalin any reasonableatmosphere. The phase space of Eq. (5) 


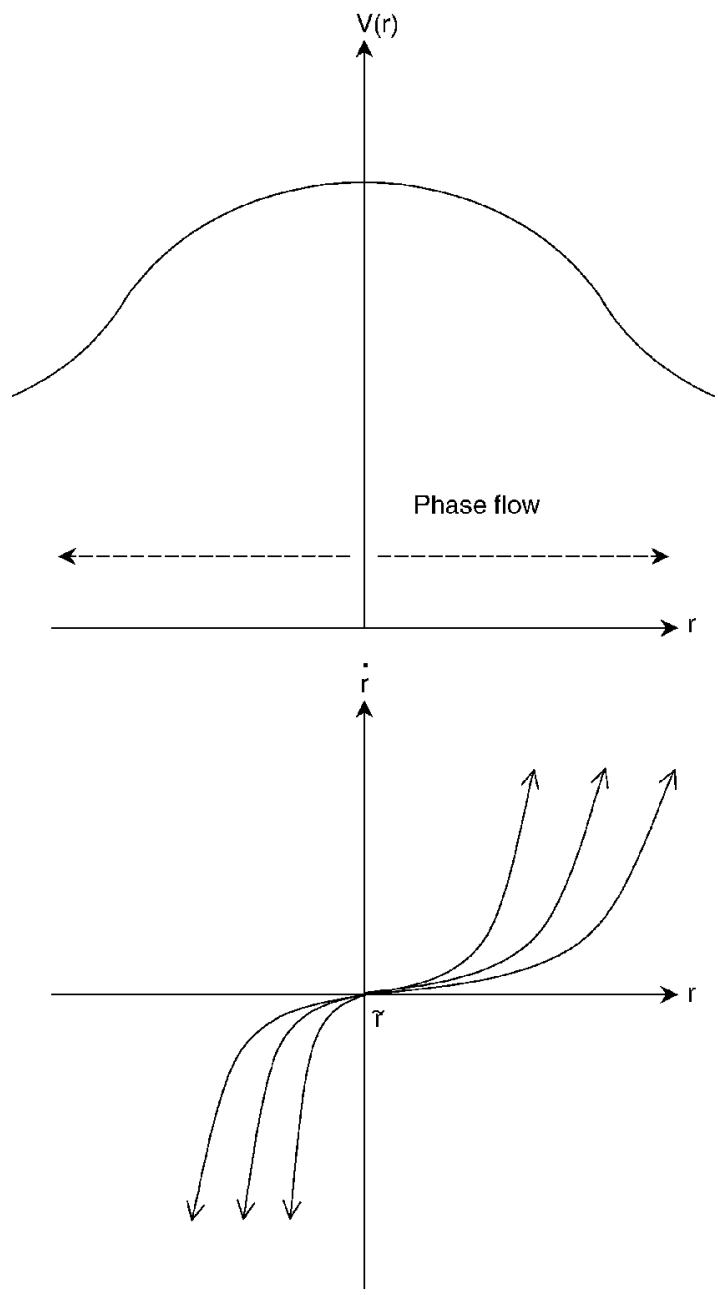

Fig. 1 Schematic potential and phase space.

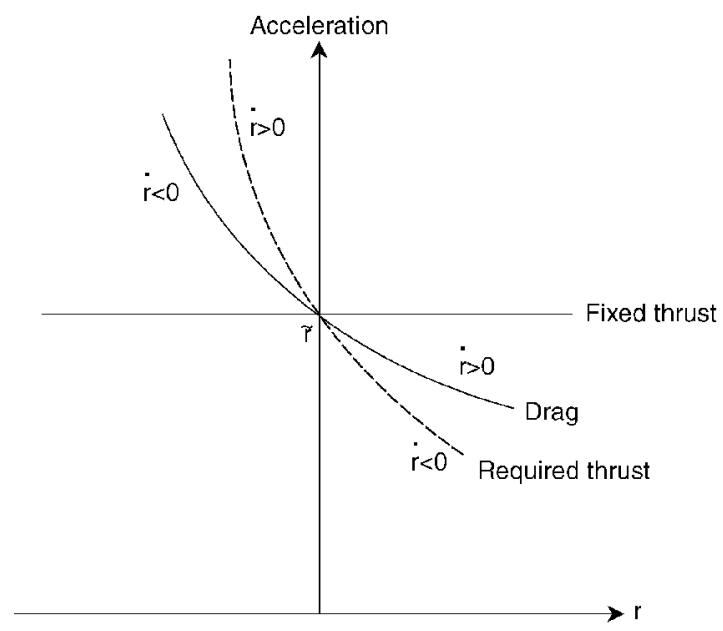

Fig. 2 Conditions for stability.

is shown schematically in Fig. 1. Note that such an unphysical atmosphere model has in fact been considered to illustrate some subtle points in optimal control theory. ${ }^{3}$

Physically, this instability is due to the interaction between the two accelerationsacting on the satellite. Figure 2 shows a schematic diagram of the variation of drag and low-thrust acceleration with altitude. For altitudes higher than the equilibrium altitude, the lowthrust acceleration exceeds the drag acceleration so that the satellite spirals outward. Conversely, for altitudes lower than the equilibrium altitude, the drag acceleration exceeds the low-thrust acceleration, and the satellite spirals inward. It can be seen that to stabilize the equilibrium operating altitude, the low-thrust acceleration would be required to be shaped as a function of altitude, as shown in Fig. 2.

\section{Nonlinear Orbit Instability}

Although it has been demonstrated that the forced circular orbit is linearly unstable, which is both a necessary and sufficient condition for nonlinear instability, it is interesting to investigate the nonlinear stability properties of Eq. (5). For ease of analysis, Eq. (5) will be considered with the exponential atmosphere model defined by Eq. (10) to obtain

$$
\frac{\mathrm{d} r}{\mathrm{~d} t}=\alpha r^{\frac{3}{2}}-\beta \sqrt{r} \exp \left(\frac{-r}{H}\right), \quad \alpha=\frac{2\|\boldsymbol{T}\|}{\sqrt{\mu} m}
$$

$$
\beta=\frac{\sqrt{\mu} C_{D} A}{m} \exp \left(\frac{R}{H}\right)
$$

This first-order dynamic system may now be written as a function of some potential $V(r)$ such that ${ }^{4}$

$$
\dot{r}=-\frac{\partial V(r)}{\partial r}
$$

where the potential is found to be

$$
V(r)=-\frac{2}{5} \alpha r^{\frac{5}{2}}-\beta H^{\frac{3}{2}} \Gamma\left[\frac{3}{2}, r / H\right]
$$

with $\Gamma$ the incomplete gamma function. It can be seen that $V(r)$ has a turning point, $V^{\prime}(r)=0$ at $r=\tilde{r}$, when

$$
\alpha / \beta=(1 / \tilde{r}) \exp (-\tilde{r} / H)
$$

which is equivalent to Eq. (6). Note that, for a given pair of parameters $\alpha$ and $\beta$, the equilibrium altitude can be determined as a solution to Eq. (16). Although implicit in $\tilde{r}$, Eq. (16) can be solved using the Lambert $W$ function (see Ref. 5), the solution of which is implemented in symbolic mathematics packages as the ProductLog function. This function is defined such that $W(z)$ returns the principal solution of $z=W e^{W}$, with $W(z)$ real if $z \succ-1 / e$, and can be viewed as an extension of the usual logarithm function. The function also satisfies the differential equation $\mathrm{d} W / \mathrm{d} z+W / z(1+W)$. When this function is used, it can be shown that Eq. (16) provides the equilibrium altitude $\tilde{r}$ as

$$
\tilde{r} / H=W(\beta / \alpha H)
$$

To demonstrate that $\tilde{r}$ is unstable in general, it must be shown that $\tilde{r}$ corresponds to a single global maximum of the potential $V(r)$. Therefore, calculating $V^{\prime \prime}(r)$ as

$$
V^{\prime \prime}(r)=-\frac{3}{2} \alpha \sqrt{r}+(\beta / 2 \sqrt{r})(1-2 r / H) \exp (-r / H)
$$

it can be seen that because $\tilde{r} \succ H / 2$ then $V^{\prime \prime}(\tilde{r}) \prec 0$ so that the equilibriumorbitradius $\tilde{r}$ correspondsto a maximum in the potential $V(r)$ and indeed $V^{\prime \prime}(r) \prec 0$ for $r \succ H / 2$. It is then sufficient to note that $V^{\prime}(r)=0$ has no solutions for $r \succ 0$, other than that defined by Eq. (16). Therefore, the equilibrium orbit radius $\tilde{r}$ corresponds to a single global maximum in the potential $V(r)$ and so the equilibrium altitude has nonlinear instability, as shown in Fig. 1.

\section{Conclusions}

It has been shown that a forced circular orbit, using a fixed lowthrust acceleration to compensate for air drag, is exponentially unstable. The instability has been determined using both a linear and nonlinear analysis. For the linear analysis, it was demonstrated that the instability exists for all reasonable atmosphere models, whereas the nonlinear analysis assumed an exponential atmosphere model. Because the linear instability condition is both necessary and sufficient, however, it is determined that the instability is independent of the atmosphere model assumed. A stable forced orbit would require a montonically increasing air density with orbit radius, which is clearly unphysical. 


\section{References}

${ }^{1}$ Roy, A. E., Orbital Motion, Adam Hilger, Bristol, England, U.K., 1987, pp. 299-331.

${ }^{2}$ Ross, I. M., and Alfriend, K. T., "Low-Earth-Orbit Maintenance: Reboost vs Thrust-Drag Cancellation," Journal of Guidance, Control, and Dynamics, Vol. 18, No. 4, 1995, pp. 930-932.

${ }^{3}$ Ross, I. M., Yan, H., and Fahroo, F., "A Curiously Outlandish Problem in Orbital Mechanics," American Astronautical Society, AAS Paper 01-430, July-Aug. 2001.

${ }^{4}$ Percival, I. C., and Richards, D., Introduction to Dynamics, Cambridge Univ. Press, Cambridge, England, U.K., 1982, pp. 1-6.

${ }^{5}$ Corless, R. M., Gonnet, G. H., Hare, D. E. G., Jeffrey, D. J., and Knuth, D. E., "On the Lambert W Function," Advances in Computational Mathematics, Vol. 5, No. 4, 1996, pp. 329-359. 